\title{
Presentation order and recognition of categorically related examples
}

\author{
DOUGLAS L. MEDIN \\ Northwestern University, Evanston, Illinois \\ and \\ JEFFREY G. BETTGER \\ Salk Institute for Biological Studies, San Diego, California
}

\begin{abstract}
Two experiments are described in which different groups of participants saw the same examples in different orders and then were given an old-new recognition test. The learning and test examples were created from different combinations from four binary-valued dimensions. One order (small change) was constructed to maximize the similarity between successive examples, and the other order (large change) minimized the similarity across successive examples. The small change condition was consistently associated with better old-new recognition than the large change condition was. These results are discussed in terms of exemplar-guided encoding and models of category generalization.
\end{abstract}

One of the more surprising aspects of research on classification learning has been the relative success of exemplar models of categorization (e.g., Allen \& Brooks, 1991; Estes, 1986; Hintzman, 1986; Medin \& Schaffer, 1978; Nosofsky, 1987). Effects of specific episodes on concept learning, problem solving, and memory are quite robust (e.g., Jacoby \& Brooks, 1984, 1989; Medin \& Ross, 1989) and provide clear counterexamples to theories which assume that abstraction is automatic and that generic memories necessarily provide the basis for performance.

Although memory for examples seems to be implicated as a factor in classification performance, it is not necessarily the case that examples are veridically encoded in a passive manner. First of all, the exemplar model that has received the most specific quantitative support, the generalized context model (Nosofsky, 1987), assumes that learners may pay differential attention to dimensions that have greater diagnostic value. If two exemplars differ only along nondiagnostic dimensions, they may not have distinct representations. The notion of differential or selective attention makes the task of contrasting the predictions of exemplar-based and rule-based models quite difficult (see, e.g., Nosofsky, Clark, \& Shin, 1989).

The present research was supported by NSF Grant BNS92-10245 to D.L.M. Brian Ross and Evan Heit provided helpful comments on an early draft of this paper. Correspondence should be addressed to D. L. Medin, Department of Psychology, Northwestern University, 2029 Sheridan Road, Evanston, IL 60208 (e-mail: medin@nwu.edu).
Brooks, Norman, and Allen (1991) found that diagnosis of skin disorders was facilitated by similar cases previously seen in the same context. Earlier examples can even influence how features of current cases are interpreted (Norman, Brooks, Coblentz, \& Babcock, 1992). In short, the encoding of examples is importantly influenced by similar prior examples (see also Ross, Perkins, \& Tenpenny, 1990).

One factor that may influence exemplar processing is the order in which instances are presented. Wattenmaker (1993) demonstrated that exemplar order affects both intentional and incidental concept learning. In particular, he studied sensitivity to within-category feature correlations when the sequence of examples was varied. In the blocked condition, examples with the same features on correlated dimensions tended to occur contiguously, whereas in the unblocked condition, examples that shared correlated features tended to be separated by intervening items. Transfer tests showed much greater sensitivity to correlated features in the blocked condition than in the unblocked condition. The blocking advantage is consistent with the idea that common or shared properties of examples that are simultaneously present in working memory are selectively strengthened (see also Elio \& Anderson, 1981).

The present studies examined the influence of order of examples on old-new recognition memory. In our basic procedure, two groups see exactly the same set of examples but in two different orders. One order maximizes and the other minimizes the similarity of successive examples. 
After the sequence of examples has been presented, the participants are given an old-new recognition test. If shared properties of successively presented examples are selectively strengthened, the two orders should yield both main effects and interactions in recognition.

Table 1 shows the two sequences of examples used in Experiment 1, represented in abstract notation. Individual examples were distinguishable from each other only with respect to the combinations of properties they manifested. For each of the constituent dimensions, one value (1) was more typical or frequent than the other (0). For the first and third dimensions, the relative frequency was 5 to 2 , and on the second and fourth dimensions, it was 4 to 3 . After participants had seen the seven examples, they were given an old-new recognition test involving seven old stimuli and nine new stimuli. The new examples consisted of the remaining possible unique combinations of binary values on the four dimensions.

The small change sequence minimized differences across successive examples. Specifically, Example 2 differed from Example 1 only along the fourth dimension; Example 3 differed from Example 2 only along the first dimension; and so on. For the large change sequence, Example 2 differed from Example 1 along three dimensions and Example 3 differed from Example 2 along two dimensions, and so on. On the average, successive examples differed on 1.2 dimensions in the small change condition and differed on 2.7 dimensions in the large change condition. This design necessarily involves a confounding of particular examples with serial position in the list. To minimize the influences of primacy and recency effects, the first and last examples were the same for each list.

The general idea is that participants ought to be able to use the just preceding example to guide the encoding of the current example in the small change condition because successive examples have numerous shared properties. Related work on effects of semantic organization on word recognition is consistent with this prediction. Specifically, when word lists comprise words from categories, recognition is better if examples are blocked by category than if they are randomly intermixed (e.g., Neely \& Balota, 1981).

Table 1

The Two Orders of Examples Used in Experiment 1

\begin{tabular}{|c|c|c|c|c|c|c|c|c|}
\hline \multirow{3}{*}{$\begin{array}{c}\text { Example } \\
\text { Order }\end{array}$} & \multicolumn{8}{|c|}{ Condition } \\
\hline & \multicolumn{4}{|c|}{ Small Change } & \multicolumn{4}{|c|}{ Large Change } \\
\hline & D1 & $\mathrm{D} 2$ & D3 & $\mathrm{D} 4$ & D1 & D2 & D3 & D4 \\
\hline 1 & 0 & 1 & 1 & 1 & 0 & 1 & 1 & 1 \\
\hline 2 & 0 & 1 & 1 & 0 & 1 & 0 & 1 & 0 \\
\hline 3 & 1 & 1 & 1 & 0 & 1 & 0 & 0 & 1 \\
\hline 4 & 1 & 0 & 1 & 0 & 1 & 1 & 1 & 0 \\
\hline 5 & 1 & 0 & 0 & 1 & 1 & 0 & 1 & 1 \\
\hline 6 & 1 & 0 & 1 & 1 & 0 & 1 & 1 & 0 \\
\hline 7 & 1 & 1 & 0 & 1 & 1 & 1 & 0 & 1 \\
\hline
\end{tabular}

Note-D1-D4 refer to dimensions. The binary notation refers to alternative values on the various dimensions.

\section{EXPERIMENT 1}

The first experiment conformed to the conditions outlined in Table 1. The stimuli were cartoon-like drawings of animals that differed in head shape (angular or round), body marking (spots or stripes), number of legs (four or eight), and tail length (long or short). As indicated in Table 1, the participants saw the seven learning examples in one of the two orders and were then given the old-new recognition test.

\section{Method}

Subjects. The subjects were 32 University of Illinois undergraduate men and women. They participated in partial fulfillment of a course requirement.

Stimuli. The cartoon-like animals in the stimulus drawings had an angular or a round head, spots or stripes, four or eight legs, and a short or a long tail. The dimensions of head shape, body marking, number of legs, and tail length corresponded to Dimensions 1-4 in Table 1. Individual stimuli differed from each other only in the combination of values they possessed. (The actual stimuli are reproduced in Medin, Wattenmaker, \& Hampson, 1987, Figure 2.) The stimuli were photocopied and organized into booklets.

Although the sequence of stimuli presented always conformed to the appropriate abstract sequence of Table 1 , the specific stimuli used to realize this design varied from subject to subject. Specifically, the assignment of specific values to the abstract notation was systematically varied so that each drawing corresponded to each abstract combination of values exactly once for the small change condition and once for the large change condition. For example, 0111 might correspond to the drawing with a round head, stripes, four legs, and a long tail for one participant and to the drawing with an angular head, stripes, four legs, and a long tail for another participant, and so on. Given that there are 16 distinct ways of assigning the combinations of values to the abstraction notation, the realization of abstract values with stimuli was exactly counterbalanced. Each participant saw all 16 stimuli: 7 old, 9 new. Note that, for each dimension, the value 1 was typical and the value 0 atypical.

Procedure. The participants were randomly assigned to either the small change or the large change condition. These two groups differed with respect to the order in which examples were presented as outlined in Table 1. All other aspects of the design and procedure were identical for the two groups.

The participants were told that they would be seeing a series of drawings of cartoon-like animals and that they would study them for a later memory test. Sets of 4-5 people were tested simultaneously. The stimuli were assembled in booklets and the participants were paced through the booklet. Specifically, people were allowed to study each page for $5 \mathrm{sec}$, followed by a 2 -sec interval where the experimenter instructed the participants to turn the page and proceed to the next study example.

A blank page appeared after the seven study examples. At this point, instructions were given for the memory test. The subjects were told that they would see more pictures, some of which had appeared before and some of which were new. They were further instructed to rate each test example on a 7-point recognition scale. The scale was designed as follows: 7 = sure that the example was old; $6=$ pretty sure the example was old $;=$ think the example is old $4=$ completely uncertain; $3=$ think the example is new; $2=$ pretty sure the example is new; $1=$ sure that it is new. The recognition test was also paced with the same 5-sec interval followed by a 2 -sec interstimulus interval. The old-new recognition test began approximately $1 \mathrm{~min}$ after the last study example had been presented.

The old-new recognition test comprised the 16 possible combinations of the binary attributes associated with head shape, body marking, number of legs, and tail length. These 16 test examples ( 7 old and 9 new) were presented in a different random order for each of the participants. 


\section{Results and Discussion}

The results showed a clear difference in old-new recognition as a function of stimulus order. Participants in the small change condition gave an average rating of 5.61 for old examples and 3.65 for new examples, producing a difference of 1.96. Participants in the large change condition averaged 5.42 for old items and 4.42 for new items, yielding a smaller difference of 1.00 . An analysis of variance (ANOVA) with the factors of groups and old versus new examples indicated a reliable groups $\times$ old versus new interaction $\left[F(1,30)=6.11, M S_{\mathrm{e}}=45.23, p<\right.$ $.05]$. The same pattern of results is obtained if ratings of 1 , 2 , or 3 are collapsed into a "new" response and 4, 5, or 6 into an "old" response.

The full breakdown of ratings for the individual items is given in Table 2 . In general, the more typical features an example possessed, the higher was its mean rating. For the new items with $0,1,2$, and 4 typical features, the mean ratings were $2.4,4.1,4.1$, and 4.9 , respectively. There are, however, some counterexamples to this typicality effect. Stimulus 0010 was rated higher than Stimulus 0011 , and Stimulus 1000 was rated higher than Stimulus 1100 (in fact, it was the highest rated new stimulus). This outcome is consistent with the idea that features shared across successive examples are differentially strengthened. Examples 2, 3, and 4 shared the pattern --10 (a " - "' indicates that the value is irrelevant), and Examples 4, 5, and 6 shared the pattern 10--). Note also that although the value 1 was typical for each dimension, the pattern --10 appeared more often than --11, and 10-appeared more often than 11--. In short, the ratings in the small change condition reflect sensitivity to conjoint frequency or correlation information. The preceding com-

Table 2

Mean Recognition Rating for Each of the 16 Possible Examples for the Small Change and Large Change Conditions in Experiment 1

\begin{tabular}{|c|c|c|c|c|c|c|}
\hline & \multicolumn{4}{|c|}{ Examples } & \multicolumn{2}{|c|}{ Condition } \\
\hline & D1 & D2 & D3 & D4 & Small Change & Large Change \\
\hline Old & $\begin{array}{l}0 \\
0 \\
1 \\
1 \\
1 \\
1 \\
1\end{array}$ & $\begin{array}{l}1 \\
1 \\
1 \\
0 \\
0 \\
0 \\
1\end{array}$ & $\begin{array}{l}1 \\
1 \\
1 \\
1 \\
0 \\
1 \\
0\end{array}$ & $\begin{array}{l}1 \\
0 \\
0 \\
0 \\
1 \\
1 \\
1\end{array}$ & $\begin{array}{c}6.38 \\
6.31 \\
6.19 \\
5.06 \\
5.18 \\
5.37 \\
4.75 \\
M=5.61\end{array}$ & $\begin{array}{c}5.75 \\
5.00 \\
5.69 \\
5.12 \\
5.69 \\
5.56 \\
5.12 \\
M=5.42\end{array}$ \\
\hline New & $\begin{array}{l}0 \\
1 \\
0 \\
0 \\
0 \\
0 \\
0 \\
1 \\
1\end{array}$ & $\begin{array}{l}0 \\
0 \\
1 \\
0 \\
0 \\
0 \\
1 \\
1 \\
1\end{array}$ & $\begin{array}{l}0 \\
0 \\
0 \\
1 \\
0 \\
1 \\
0 \\
0 \\
1\end{array}$ & $\begin{array}{l}0 \\
0 \\
0 \\
0 \\
1 \\
1 \\
1 \\
0 \\
1\end{array}$ & $\begin{array}{c}2.12 \\
5.06 \\
2.37 \\
3.56 \\
3.38 \\
2.94 \\
4.12 \\
4.56 \\
4.62 \\
M=3.65\end{array}$ & $\begin{array}{c}2.75 \\
4.69 \\
4.56 \\
4.75 \\
4.25 \\
4.12 \\
3.86 \\
5.06 \\
5.12 \\
M=4.42\end{array}$ \\
\hline
\end{tabular}

Note-D1-D4 refer to dimensions. The binary values refer to alternative values on the dimensions.
Table 3

The Two Orders of Examples Used in the Second Experiment Presented in Abstract Notation

\begin{tabular}{|c|c|c|c|c|c|c|c|c|}
\hline \multirow{3}{*}{$\begin{array}{c}\text { Example } \\
\text { Order }\end{array}$} & \multicolumn{8}{|c|}{ Condition } \\
\hline & \multicolumn{4}{|c|}{ Small Change } & \multicolumn{4}{|c|}{ Large Change } \\
\hline & $\overline{\mathrm{D} 1}$ & D2 & D3 & D4 & D1 & D2 & D3 & D4 \\
\hline 1 & 1 & 1 & 1 & 0 & 1 & 1 & 1 & 0 \\
\hline 2 & 0 & 1 & 1 & 0 & 1 & 0 & 0 & 1 \\
\hline 3 & 0 & 1 & 1 & 1 & 0 & 1 & 1 & 1 \\
\hline 4 & 1 & 0 & 1 & 1 & 1 & 0 & 1 & 0 \\
\hline 5 & 1 & 0 & 1 & 0 & 1 & 1 & 0 & 1 \\
\hline 6 & 1 & 0 & 0 & 1 & 1 & 0 & 1 & 1 \\
\hline 7 & 1 & 1 & 0 & 1 & 0 & 1 & 1 & 0 \\
\hline 8 & 1 & 1 & 1 & 1 & 1 & 1 & 1 & 1 \\
\hline
\end{tabular}

Note-D1-D4 refer to dimensions. The binary values refer to alternative values on the dimensions.

parisons, which pit typicality against conjoint frequency, are suggestive, but they fall short of statistical reliability. The large change condition also yields some exceptions for typicality ordering, but they are not systematic.

An ANOVA with the factors of groups and stimuli yielded a main effect of stimuli $[F(15,450)=4.32$, $\left.M S_{\mathrm{e}}=3.46, p<.001\right]$ and a stimuli $\times$ groups interaction $\left[F(15,450)=1.97, M S_{\mathrm{e}}=3.46, p<.05\right]$. Separate analyses of old and new stimuli revealed no reliable effect for old examples, but for the new stimuli there was a main effect of groups $\left[F(1,38)=5.18, M S_{\mathrm{e}}=\right.$ $8.40, p<.05]$ and of Stimuli $\left[F(8,240)=2.86, M S_{\mathrm{e}}=\right.$ $3.70, p<.01]$ as well as a marginal groups $\times$ stimuli interaction $\left[F(8,240)=1.77, M S_{\mathrm{e}}=3.70, p<.10\right]$.

The advantage from the small change condition agrees with the general idea of exemplar-guided encoding described earlier. The results are also consistent with the idea that features shared by successive training examples are preferentially strengthened. Experiment 2 takes a more systematic look at the role played by overlapping patterns in recognition.

\section{EXPERIMENT 2}

The second experiment used the same set of drawings as did the first, but the sequence of examples was different. The orders used for the small change and large change conditions are shown in abstract notation in Table 3. Eight examples were presented, and again the first and last stimulus were the same for the two groups. For the small change condition, successive examples differed on an average of 1.3 dimensions, and for the large change condition, examples differed on an average of 2.7 dimensions.

Our primary focus was on main effects of order but, in addition, we anticipated a stimulus $\times$ order interaction. For the small change condition, Training Examples 4, 5, and 6 had an overlapping pattern of 10--, which was matched by new Stimulus 1000 . New Stimulus 1100 had more typical features than did 1000 and the pattern 11-appeared as often as 10--; but the three instances of 11-were not clustered. In the large change condition, the pattern 10-- did not even appear twice in succession. In brief, 
we expected that the small change (but not the large change) condition would yield higher familiarity ratings for Stimulus 1000 than for Stimulus 1100 .

\section{Method}

Subjects. The subjects were 32 University of Illinois undergraduate men and women. They participated in partial fulfillment of a course requirement.

Stimuli and Procedure. The cartoon-like animals used in Experiment 1 were employed again. The procedures were identical to those of Experiment 1, with a single exception: the sequence of examples during the study period followed the abstract structure shown in Table 3 rather than that of Table 1 . In other words, eight rather than seven training examples were given and two new orderings were created.

\section{Results and Discussion}

The old-new recognition task revealed both a main effect of sequence and an interaction of sequence with particular examples. Again, this pattern holds for mean ratings as well as collapsed "new" versus "old" judgments. The small change group gave old patterns an average rating of 6.11 and new examples an average rating of 3.90 , producing a difference of 2.21 . The large change group gave old patterns an average rating of 5.45 and new examples a rating of 4.68 , yielding a substantially smaller difference of 0.77 . In brief, old-new recognition was better for the small change group than for the large change group. An ANOVA with the factors of groups and old versus new revealed a significant interaction $[F(1,30)=$ $\left.8.06, M S_{\mathrm{e}}=66.4, p<.01\right]$.

The mean ratings for the two groups are given in Table 4 . First of all, one may note that the more typical values an example possessed (the more 1s it had), the higher was its rating. The overall mean ratings for 0,1 , 2,3 , and 4 typical values were $3.75,4.08,5.14,5.85$, and 6.37 , respectively.

Table 4

Mean Recognition Rating for Each of the 16 Possible Examples for the Small Change and Large Change Conditions in Experiment 1

\begin{tabular}{cccccccc}
\hline & \multicolumn{3}{c}{ Examples } & & \multicolumn{2}{c}{ Condition } \\
\cline { 2 - 4 } & D1 & D2 & D3 & D4 & & Small Change & Large Change \\
\hline Old & 1 & 1 & 1 & 0 & & 6.50 & 5.31 \\
& 0 & 1 & 1 & 0 & & 6.00 & 4.12 \\
& 0 & 1 & 1 & 1 & & 5.94 & 5.12 \\
& 1 & 0 & 1 & 1 & & 5.94 & 5.94 \\
& 1 & 0 & 1 & 0 & & 6.18 & 5.00 \\
& 1 & 0 & 0 & 1 & & 5.75 & 5.88 \\
& 1 & 1 & 0 & 1 & & 6.00 & 5.06 \\
& 1 & 1 & 1 & 1 & & 6.62 & 6.12 \\
& & & & & $M=6.11$ & $M=5.45$ \\
& & & & & & 3.25 & 4.25 \\
& 1 & 0 & 0 & 0 & & 5.50 & 4.25 \\
& 0 & 1 & 0 & 0 & & 2.50 & 3.81 \\
& 0 & 0 & 1 & 0 & 3.81 & 4.88 \\
& 0 & 0 & 0 & 1 & 2.88 & 4.81 \\
0 & 0 & 1 & 1 & 4.81 & 5.19 \\
0 & 1 & 0 & 1 & 4.00 & 5.38 \\
1 & 1 & 0 & 0 & & 4.44 & 4.81 \\
& & & & $M=3.90$ & $M=4.68$
\end{tabular}

Note-D1-D4 refer to dimensions. The binary values refer to alternative values on the dimensions.
In addition to the main effect of small change versus large change, information order also interacted with particular stimuli. Ratings of new stimuli by the large change group were quite consistent with typicality, especially if one keeps in mind potential differences in the salience of dimensions. In the small change condition, there was a notable exception to typicality ordering. As predicted, Stimulus 1000 received a higher rating than did Stimulus $1100[t(15)=2.41, p<.05]$. Note that this difference cannot be explained in terms of either dimensional salience (the patterns only differ on the second dimension and Stimulus 1100 has the typical value) and conjoint frequency (10-- and 11-- occur equally often). Instead the results suggest that the clustering of correlated features is the key factor.

Statistical tests supplement the preceding picture. An ANOVA with the factors of groups and stimuli indicated a significant effect for stimuli $\left[F(15,450)=7.31, M S_{\mathrm{e}}=\right.$ $3.74, p<.001]$ and a reliable effect of groups $[F(1,30)=$ $\left.5.69, M S_{\mathrm{e}}=5.68, p<.05\right]$. A corresponding analysis of new stimuli indicated a reliable effect of stimuli $\left[F(7,210)=3.32, M S_{\mathrm{e}}=3.89, p<.01\right]$ and a marginal groups $\times$ stimuli interaction $\left[F(7,210)=1.91, M S_{\mathrm{e}}=\right.$ $3.89, p=.07]$. To compare the response to the new Stimulus 1000 in the two groups, the mean rating to the other new stimuli was subtracted from the rating of Stimulus 1000 for each subject. For the small change group, this difference averaged 1.86; the difference was -.049 for the large change group. This difference between groups was reliable $[t(30)=3.69, p<.01]$.

In summary, the results again provide general support for exemplar-guided encoding where shared properties of successive examples are differentially strengthened. The small change condition had much greater overlap of successive examples and yielded reliably better old-new recognition than did the large change condition. On a more detailed level, the results support the idea that shared properties of successive examples are differentially strengthened. The new example receiving the highest familiarity rating (1000) was not the stimulus that comprised the most frequent or typical features but rather the one that was consistent with the configuration of shared features of successive examples (10--).

\section{GENERAL DISCUSSION}

Our central result is that the order in which a set of examples is presented has a major influence on old-new recognition. Specifically, oldnew recognition for a set of examples is reliably better if the successive examples are as similar as possible than if the successive examples are as different as possible. This finding held in both of the present experiments, and in other unpublished studies, using different sequences and stimuli, we have replicated this main result.

The differences between the two groups are striking in that both groups saw the same examples over the same short time period (less than $1 \mathrm{~min}$ ), with the first and last examples being in the same order. Yet there were large differences on the old-new recognition test given only a minute later.

The other main result concerns patterns of false alarms (familiarity ratings) to new patterns. New stimuli consistent with combinations of features shared by successive learning items receive higher familiarity 
ratings than would be predicted by the frequency of their individual constituent features. Our recognition results parallel Wattenmaker's (1993) findings on blocked presentation and categorization, as well as other results showing the advantage of blocked over random presentation of categorized word lists (Neely \& Balota, 1981).

In their most straightforward form, exemplar models of categorization cannot account for the effect of information order. If each example is encoded and stored separately, memory should be a function only of the set of examples seen and of retention conditions (see Whittlesea, 1987, for related observations). For both the small change and large change orders, however, the same set of examples was presented and the retention interval was the same for the two groups.

Our data also have implications for exemplar models that allow for selective attention, such as the Medin and Schaffer (1978) context model. Both the original context model and its generalization by Nosofsky (1987) assume selective attention at the level of dimensions such that some dimensions may be weighted more than others. The present results suggest selectivity at the level of specific stimuli, favoring features that are shared across successive examples (see also Medin \& Edelson, 1988; Medin \& Florian, 1992).

The present results are not specifically tied to exemplar models of categorization. For example, they are compatible with the Anderson, Kline, and Beasley (1979) ACT generalization model. ACT assumes that generalizations are formed on the basis of shared properties of items in working memory and, with some appropriate extensions, might readily be applied to our experimental situation.

Whether the results generalize to the use of stimuli that do not comprise different combinations of values from a small set of dimensions or stimuli that do not have a typicality structure is an open question. On the other hand, it is precisely stimuli of the character of those used in the present studies that have produced some of the clearest evidence favoring exemplar models of classification (e.g., Medin \& Schaffer, 1978). Our results, overall, are compatible with exemplar-guided encoding, but the products of such codings reflect attentional processes favoring some values (shared values) over others.

\section{REFERENCES}

AlLEN, S. W., \& Brooks, L. R. (1991). Specializing the operation of an explicit rule. Journal of Experimental Psychology: General, 120, 3-19.

Anderson, J. R., Kline, P. J., \& Beasley, C. M. (1979). A general learning theory and its application to schema abstraction. In G. H. Bower (Ed.), The psychology of learning and motivation (Vol. 13, pp. 227-319). New York: Academic Press.

Brooks, L. R., Norman, G. R., \& Allen, S. W. (1991). Role of specific similarity in a medical diagnosis task. Journal of Experimental Psychology: General, 120, 278-287.

Elio, R., \& ANDERSON, J. R. (1981). Effects of category generalizations and instance similarity on schema abstraction. Journal of Experimental Psychology: Human Learning \& Memory, 7, 397-417.
EsTES, W. K. (1986). Memory storage and retrieval processes in category learning. Journal of Experimental Psychology: General, 115, 155-175.

Hintzman, D. L. (1986). "Schema abstraction" in a multiple-trace memory model. Psychological Review, 93, 411-428.

JACOBY, L. L., \& BROOKs, L. R. (1984). Non-analytic cognition: Memory, perception, and concept learning. In G. H. Bower (Ed.), The psychology of learning and motivation (Vol. 18, pp. 1-47). New York: Academic Press.

Medin, D. L., \& Edelson, S. E. (1988). Problem structure and the use of base rate information from experience. Journal of Experimental Psychology: General, 117, 68-85.

Medin, D. L., \& Florian, J. E. (1992). Abstraction and selective coding in exemplar-based models of categorization. In A. F. Healy, S. M. Kosslyn, \& M. Shiffrin (Eds.), From learning processes to cognitive processes: Essays in honor of William K. Estes (Vol. 2, pp. 207-234). Hillsdale, NJ: Erlbaum.

Medin, D. L., \& Ross, B. H. (1989). The specific character of abstract thought: Categorization, problem-solving, and induction. In R. J. Sternberg (Ed.), Advances in the psychology of human intelligence (Vol. 5, pp. 189-223). Hillsdale, NJ: Erlbaum.

Medin, D. L., \& SChafFer, M. M. (1978). A context theory of classification learning. Psychological Review, 85, 207-238.

Medin, D. L., WatTenmaker, W. D., \& Hampson, S. E. (1987). Family resemblance, concept cohesiveness, and category structure. Cognitive Psychology, 19, 242-279.

NeELy, J. H., \& BALOTA, D. A. (1981). Test-expectancy and semanticorganization effects in recall and recognition. Memory \& Cognition, 9, 283-300.

Norman, G. R., Brooks, L. R., Coblentz, C. L., \& Babcock, C. J. (1992). The correlation of feature identification and category judgments in diagnostic radiology. Memory \& Cognition, 20, 344-355.

Nosofsky, R. M. (1987). Attention and learning processes in the identification and categorization of integral stimuli. Joumal of Experimental Psychology: Learning, Memory, \& Cognition, 14, 700-708.

Nosofsky, R. M., Clark, S. E., \& SHIN, J. H. (1989). Rules and exemplars in categorization, identification, and recognition. Journal of Experimental Psychology: Learning, Memory, \& Cognition, 15, 282-304.

Ross, B. H., Perkins, S. J., \& Tenpenny, P. L. (1990). Remindingbased category learning. Cognitive Psychology, 22, 460-492.

WATtENMAKER, W. D. (1993). Incidental concept learning, feature frequency and correlated properties. Journal of Experimental Psychology: Human Learning \& Memory, 19, 203-222.

Whit lesea, B. W. A. (1987). Preservation of specific experiences in the representation of general knowledge. Journal of Experimental Psychology: Learning, Memory, \& Cognition, 13, 3-17.

(Manuscript received August 4, 1993; revision accepted for publication December 1, 1993.) 